\title{
Some remarks on Radon-Nikodým compact spaces
}

\author{
by
}

Alexander D. Arvanitakis (Athens)

\begin{abstract}
The class of quasi Radon-Nikodým compact spaces is introduced. We prove that this class is closed under countable products and continuous images. It includes the Radon-Nikodým compact spaces. Adapting Alster's proof we show that every quasi Radon-Nikodým and Corson compact space is Eberlein. This generalizes earlier results by J. Orihuela, W. Schachermayer, M. Valdivia and C. Stegall. Further the class of almost totally disconnected spaces is defined and it is shown that every quasi Radon-Nikodým space which is almost totally disconnected is actually a Radon-Nikodým compact space embeddable in the space of probability measures on a scattered compact space.
\end{abstract}

1. Introduction. We call a compact space $K$ quasi Radon-Nikodým if there exists a lower semicontinuous (as a function on $K \times K$ ) fragmenting quasimetric defined on $K$. By a quasimetric, we mean a "metric" failing to satisfy the triangle inequality, i.e. a function $f: K \times K \rightarrow[0,1]$ for which:

1. For all $x, y \in K, f(x, y)=0 \Leftrightarrow x=y$.

2. For all $x, y \in K, f(x, y)=f(y, x)$.

In Propositions 3.2 and 3.3, we show that this class of spaces is stable. More precisely, continuous images, closed subspaces and countable Cartesian products of quasi Radon-Nikodým compact spaces are also quasi RadonNikodým. In addition, by Proposition 3.4, quasi Radon-Nikodým compact spaces are fragmented by a metric. This class is closely related to the well known class of Radon-Nikodým compact spaces, introduced by O. Reynov [11] and independently by I. Namioka [9]. We recall that by a well known characterization due to I. Namioka [9], a compact space $K$ is Radon-Nikodým if and only if there exists a lower semicontinuous fragmenting metric on $K$. Thus Radon-Nikodým compact spaces are quasi Radon-Nikodým. Moreover this remains valid for their continuous images. Actually Theorem 3.6 yields

2000 Mathematics Subject Classification: Primary 46B22, 46B26; Secondary 54G12.

Key words and phrases: Radon-Nikodým compact, Corson compact, Eberlein compact, scattered compact. 
that every totally disconnected quasi Radon-Nikodým space is RadonNikodým and further it is homeomorphic to a closed subset of a countable product of scattered compact spaces. This extends a result due to E. Reznichenko, which asserts the latter for Radon-Nikodým compact spaces.

As a consequence, we deduce that continuous images of Radon-Nikodým compact spaces are also Radon-Nikodým, provided that they are totally disconnected. This result is due to S. Argyros [4], and with a different proof is contained in [7].

Thus the two classes are identical when restricted to totally disconnected compact spaces.

In Proposition 3.9 we derive a characterization of continuous images of totally disconnected Radon-Nikodým spaces. As a consequence, we obtain an equivalent reformulation of the problem whether or not continuous images of Radon-Nikodým compact spaces remain Radon-Nikodým (Proposition 3.10).

Section 4 is devoted to the proof that a quasi Radon-Nikodým compact space is Eberlein, provided that it is Corson. This in particular implies a well known result which has been proved by J. Orihuela, W. Schachermayer and M. Valdivia [10] and independently by C. Stegall [14], namely that a Corson Radon-Nikodým compact space is Eberlein.

Our approach to proving this result is different from those used by the above mentioned authors. We adapt Alster's techniques from [1]. We recall that Alster [1] proved that every scattered Corson compact space is strong Eberlein.

There are several common points between the quasi Radon-Nikodým compact spaces and the scattered ones. Roughly speaking, in our approach, we handle pairs of disjoint closed subsets $\left(K_{i}^{0}, K_{i}^{1}\right)_{i}$ with $f$-distance greater than $\varepsilon>0$ where $f$ is the lower semicontinuous fragmenting quasimetric, in a similar manner to what Alster did for clopen subsets of scattered spaces. Also we handle open subsets with $f$-diameter less than $\varepsilon$ similarly to points of scattered spaces.

In Section 5 we introduce almost totally disconnected compact spaces. A compact space $K$ is called almost totally disconnected if it can be embedded in $[0,1]^{\Gamma}$ for some set $\Gamma$ in such a way that for every $k \in K$, the set

$$
\{\gamma \in \Gamma: k(\gamma) \neq 0,1\}
$$

is at most countable.

These spaces strictly generalize both Corson and totally disconnected compact spaces. For example the long line which belongs to none of the above two classes is almost totally disconnected.

We prove that 
TheOREM 1.1. An almost totally disconnected quasi Radon-Nikodým compact space can be embedded in the space of regular Borel probability measures on some scattered compact space.

The proof requires techniques introduced in Section 4 and moreover we adopt techniques from [5], related to the well known Ditor Theorem ([6]). The latter states that for every compact $K$, there exists a totally disconnected compact space $L$ with the same topological weight as $K$, mapping continuously onto $K$ by a map that admits a regular averaging operator.

During the preparation of the paper, Professor I. Namioka informed us about the relation between the class of quasi Radon-Nikodým compact spaces and that of strongly fragmented compact spaces. Let us recall the definition of the latter: A metric $d$ defined on a compact space $K$ is called a Reznichenko metric if for any two distinct elements $x, y$ of $K$, there are open neighborhoods $O_{x}, O_{y}$ of $x$ and $y$ respectively such that

$$
d\left(O_{x}, O_{y}\right)=\inf \left\{d\left(x^{\prime}, y^{\prime}\right): x^{\prime} \in O_{x}, y^{\prime} \in O_{y}\right\}>0 .
$$

Following A. Arkhangel'skiu [3, p. 104] a compact space $K$ is called strongly fragmented if there exists a Reznichenko metric defined on $K$ that fragments $K$.

As was pointed out to us by Professor I. Namioka, the class of quasi Radon-Nikodým compact spaces, introduced here, and the class of strongly fragmented compact spaces are identical. This was proved by B. Cascales, E. Matoušková, I. Namioka and J. Orihuela.

Acknowledgements. I would like to thank Professor S. Argyros for his invaluable help during the preparation of this paper and also Professors B. Cascales, E. Matoušková, I. Namioka and J. Orihuela for their permission to mention here the above result.

2. Notation and definitions. In what follows, all spaces are assumed to be compact and Hausdorff. For a set $A$, we denote by $|A|$ the cardinality of $A$. We will need the definitions of the following classes of compact spaces:

Definition 2.1. A compact space $K$ is called Eberlein if it can be embedded as a weakly compact subset in a Banach space.

D. Amir and J. Lindenstrauss in the well known paper [2] have characterized Eberlein compact spaces as those compact spaces that can be embedded as weakly compact subsets in $c_{0}(\Gamma)$ for some set $\Gamma$.

This characterization gives rise to the following generalization:

Definition 2.2. A compact space $K$ is called Corson if it embeds in

$$
\Sigma\left([0,1]^{\Gamma}\right)=\left\{x \in[0,1]^{\Gamma}:|\{\gamma \in \Gamma: x(\gamma) \neq 0\}| \leq \aleph_{0}\right\} .
$$


Since for the weak* topology a definition analogous to that of Eberlein compact spaces would be vacuous (any compact space can be embedded in $\left.\left(C(K)^{*}, w^{*}\right)\right)$, we restrict ourselves to the duals of Asplund spaces:

Definition 2.3. A compact space $K$ is called Radon-Nikodým if it embeds in the dual of an Asplund space equipped with the weak* topology.

For a set $K$, a map $f: K \times K \rightarrow[0,1]$ is called a quasimetric if:

1. For all $x, y \in K, f(x, y)=0 \Leftrightarrow x=y$.

2. For all $x, y \in K, f(x, y)=f(y, x)$.

In other words a quasimetric is a metric failing the triangle inequality.

Hereafter, for a quasimetric $f$, a subset of $M$ of $K$ and an $\varepsilon>0$, we shall denote by $B_{f}(M, \varepsilon)$ the set

$$
\{x \in K: \exists y \in M: f(x, y) \leq \varepsilon\} .
$$

Let $K$ be a compact space and $f: K \times K \rightarrow[0,1]$ be a quasimetric on $K$ (which need not have any relation with the topology of $K$ ). We say that $f$ fragments $K$ if for every $\varepsilon>0$ and for every closed subset $M$ of $K$ (or equivalently, by Zorn's Lemma, for every subset of $K$ ) there exists a relatively open and non-empty subset $V$ of $M$ such that

$$
f \text {-diam }(V)=\sup \{f(x, y): x, y \in V\}<\varepsilon .
$$

Using the same notation, we say that $f$ is lower semicontinuous if it is lower semicontinuous as a map on $K \times K$ (i.e. for each $a \in[0,1]$ the set $\{(x, y) \in K \times K: f(x, y) \leq a\}$ is closed in $K \times K)$.

For convenience, we shall use the following notation: For $W_{1}, W_{2} \subset K$ we denote by $f\left(W_{1}, W_{2}\right)$ the real number

$$
\inf \left\{f(x, y): x \in W_{1}, y \in W_{2}\right\} .
$$

I. Namioka [9] has proved the following simple and useful characterization of Radon-Nikodým compact spaces:

Theorem 2.1. A compact space $K$ is Radon-Nikodým if and only if there exists a lower semicontinuous fragmenting metric on $K$.

Using this characterization, it is not difficult to prove the following:

Any scattered compact space $K$ is Radon-Nikodým, the lower semicontinuous fragmenting metric on $K$ being the discrete metric. (Being Hausdorff implies the lower semicontinuity in this case.)

Any Eberlein compact space $K$ is Radon-Nikodým, the lower semicontinuous fragmenting metric on $K$ being the norm of $c_{0}(\Gamma)$.

Also it is not difficult to prove, using the same characterization, that the class of Radon-Nikodým compact spaces is closed under taking countable 
products and closed subspaces. Thus a closed subspace of a countable product of scattered compact spaces is Radon Nikodým and, as we shall see, every totally disconnected Radon-Nikodým compact space is of this kind.

Theorem 2.1 also gives rise to the following definition:

Definition 2.4. A compact space $K$ is called quasi Radon-Nikodým if there exists a lower semicontinuous fragmenting quasimetric on $K$.

In what follows, for a subspace $L$ of the cube $[0,1]^{\Gamma}$ and $A \subset \Gamma$, we denote by $\pi_{A}$ the projection of $L$ to the $A$ coordinates. For $\gamma \in \Gamma$ we shall also write $\pi_{\gamma}$ instead of $\pi_{\{\gamma\}}$.

\section{Quasi Radon-Nikodým compact spaces}

Lemma 3.1. Assume $K$ a Hausdorff compact space, and $f: K \times K \rightarrow[0,1]$ a lower semicontinuous quasimetric on $K$. Assume moreover that $F_{1}, F_{2}$ are disjoint closed subsets of $K$. Then

1. $f\left(F_{1}, F_{2}\right)>0$.

2. If $f\left(F_{1}, F_{2}\right)>\varepsilon$ for some $\varepsilon>0$, then there are open subsets $U, V$ of $K$ such that $F_{1} \subset U, F_{2} \subset V$ and $f(U, V)>\varepsilon$.

Proof. 1. Assume on the contrary that $f\left(F_{1}, F_{2}\right)=0$, and for each $n \in \mathbb{N}$ take $x_{n} \in F_{1}, y_{n} \in F_{2}$ such that $f\left(x_{n}, y_{n}\right)<1 / n$. We can pass to subnets of $\left\{x_{n}\right\}_{n \in \mathbb{N}}$ and $\left\{y_{n}\right\}_{n \in \mathbb{N}}$ converging to $x \in F_{1}$ and $y \in F_{2}$ respectively. This is a contradiction, since by the lower semicontinuity and the separating property of $f$, there are open sets $V_{1} \ni x$ and $V_{2} \ni y$ such that $f\left(V_{1}, V_{2}\right)>0$.

2. Fix $y \in F_{2}$. Using the compactness of $F_{1}$, we can find an open cover $U_{1}^{y}, \ldots, U_{n}^{y}$ of $F_{1}$ and open sets $V_{1}^{y}, \ldots, V_{n}^{y}$ containing $y$ such that $f\left(U_{i}^{y}, V_{i}^{y}\right)>\varepsilon$. Then $U^{y}=\bigcup_{i=1}^{n} U_{i}$ contains $F_{1}, V^{y}=\bigcap_{i=1}^{n} V_{i}$ contains $y$, and $f\left(U^{y}, V^{y}\right)>\varepsilon$. Repeating the same argument using the compactness of $F_{2}$, we can find $U_{1}, \ldots, U_{n}$ containing $F_{1}$ and $V_{1}, \ldots, V_{n}$ covering $F_{2}$ such that $f\left(U_{i}, V_{i}\right)>\varepsilon$. Then $U=\bigcap_{i=1}^{n} U_{i}$ contains $F_{1}, V=\bigcup_{i=1}^{n} V_{i}$ contains $F_{2}$ and $f(U, V)>\varepsilon$.

Proposition 3.2. The continuous image of a quasi Radon-Nikodým compact space is also quasi Radon-Nikodým.

Proof. Let $K$ be a quasi Radon-Nikodým compact space, $L$ a Hausdorff compact space, and $\phi: K \rightarrow L$ a continuous onto map. Let also $f: K \times K \rightarrow$ $[0,1]$ be a lower semicontinuous fragmenting quasimetric for $K$, and define $g: L \times L \rightarrow[0,1]$ by $g(x, y)=f\left(\phi^{-1}(x), \phi^{-1}(y)\right)$. It suffices to show that $g$ is a lower semicontinuous fragmenting quasimetric. By Lemma 3.1, $g$ is clearly a quasimetric. To see that it is fragmenting, choose $M \subset L$ closed and $\varepsilon>0$. By Zorn's Lemma find $N \subset K$ closed such that $\phi \mid N: N \rightarrow M$ is onto and irreducible (i.e. for every $N^{\prime} \subset N$ closed, $\phi \mid N^{\prime}$ is not onto $M$ ). By 
the fragmentability of $f$, there exists $\emptyset \neq V \subset N$ relatively open such that $f$-diam $(V)<\varepsilon$, and by the irreducibility of $\phi \mid N$, it follows that $\phi(V)$ has non-empty interior in $M$. Since for every $x, y \in \phi(V), \phi^{-1}(x) \cap V \neq \emptyset$ and $\phi^{-1}(y) \cap V \neq \emptyset$, it follows that $g(x, y)<\varepsilon$.

To see that $g$ is lower semicontinuous, let $x, y \in L$ and $g(x, y)>\varepsilon$. Since $g(x, y)=f\left(\phi^{-1}(x), \phi^{-1}(y)\right)$, by Lemma 3.1 and the lower semicontinuity of $f$, there exist open sets $G_{1} \supset \phi^{-1}(x)$ and $G_{2} \supset \phi^{-1}(y)$ such that $f\left(G_{1}, G_{2}\right)>\varepsilon$. Let $\left\{U_{i}\right\}_{i \in I},\left\{V_{i}\right\}_{i \in I}$ be neighborhood bases of $x$ and $y$ respectively. Since $\bigcap_{i \in I} \bar{U}_{i}=\{x\}$ and $\bigcap_{i \in I} \bar{V}_{i}=\{y\}$, we get $\bigcap_{i \in I} \phi^{-1}\left(\bar{U}_{i}\right)$ $\subset G_{1}$ and $\bigcap_{i \in I} \phi^{-1}\left(\bar{V}_{i}\right) \subset G_{2}$. By the compactness of $K$, there are open sets $U$ and $V$ containing $x$ and $y$ respectively such that $\phi^{-1}(U) \subset G_{1}$ and $\phi^{-1}(V) \subset G_{2}$. Consequently, $g(U, V)>\varepsilon$ and the proof is complete.

REMARK 3.1. Since a Radon-Nikodým compact space is also quasi Radon-Nikodým, we deduce that continuous images of Radon-Nikodým compact spaces are quasi Radon-Nikodým.

REMARK 3.2. Letting in the previous proposition $f$ be the discrete metric on $K$ (in this case $K$ is scattered), we infer that $g$ is also a discrete metric on $L$, thus showing that a continuous image of a scattered compact space is also scattered.

Proposition 3.3. A closed subspace of a quasi Radon-Nikodým compact space is also quasi Radon-Nikodým. The Cartesian product of countably many quasi Radon-Nikodým compact spaces is quasi Radon-Nikodým.

Proof. The first assertion is obvious, since a lower semicontinuous fragmenting quasimetric, restricted to a closed subspace, remains a lower semicontinuous fragmenting quasimetric.

For the second statement, let $K_{n}, n \in \mathbb{N}$, be a countable family of quasi Radon-Nikodým spaces, and $f_{n}: K_{n} \times K_{n} \rightarrow[0,1]$ be a lower semicontinuous fragmenting quasimetric on $K_{n}$. Set $K=\prod_{n \in \mathbb{N}} K_{n}$ and define $f: K \times K \rightarrow[0,1]$ by

$$
f(x, y)=\sum_{n=1}^{\infty} \frac{1}{2^{n}} f_{n}\left(\pi_{n}(x), \pi_{n}(y)\right) .
$$

We will show that $f$ is a lower semicontinuous fragmenting quasimetric on $K$.

It is clearly a quasimetric on $K$. To see that it is lower semicontinuous, let $x=\left(x_{n}\right)_{n \in \mathbb{N}}, y=\left(y_{n}\right)_{n \in \mathbb{N}} \in K$ and assume that $f(x, y)>\varepsilon$. Then for some $k \in \mathbb{N}$,

$$
\delta=\sum_{n=1}^{k} \frac{1}{2^{n}} f_{n}\left(x_{n}, y_{n}\right)-\varepsilon>0 .
$$


By the lower semicontinuity of $f_{n}$, find now $U_{n}, V_{n} \subset K_{n}$ open such that $x_{n} \in U_{n}, y_{n} \in V_{n}$ and $f_{n}\left(U_{n}, V_{n}\right)>f_{n}\left(x_{n}, y_{n}\right)-\delta / k$. It can be easily checked that setting

$$
\begin{aligned}
& U=\left\{x \in K: \pi_{n}(x) \in U_{n}, 1 \leq n \leq k\right\}, \\
& V=\left\{z \in K: \pi_{n}(z) \in V_{n}, 1 \leq n \leq k\right\},
\end{aligned}
$$

we have $x \in U, y \in V$ and $f(U, V)>\varepsilon$.

Let us prove that $f$ is also fragmenting. Let $F$ be a subset of $K$ and $\varepsilon>0$. There exists a $k \in \mathbb{N}$ such that $\sum_{n=k+1}^{\infty} 2^{-n}<\varepsilon / 2$. We define inductively for each $1 \leq m \leq k$ an open subset $U_{m}$ of $\prod_{n=1}^{m} K_{n}$ such that $\pi_{\{1, \ldots, m\}}(F) \cap U_{m}$ is non-empty and has $\sum_{n=1}^{m} 2^{-n} f_{n}$-diameter less than $\sum_{n=1}^{m} 2^{-n} \varepsilon / 2$. Assume $U_{m}$ has been defined. Then $F \cap \pi_{\{1, \ldots, m\}}^{-1}\left(U_{m}\right)$ is non-empty and hence $W=\pi_{m+1}\left(F \cap \pi_{\{1, \ldots, m\}}^{-1}\left(U_{m}\right)\right)$ has a relatively open subset of $f_{m+1}$-diameter less than $\varepsilon / 2$. Let $V_{m+1}$ be an open subset of $K_{m+1}$ such that $W \cap V_{m+1} \neq \emptyset$ and $f_{m+1}-\operatorname{diam}\left(W \cap V_{m+1}\right)<\varepsilon / 2$. Then

$$
U_{m+1}=\left\{x=\left(x_{n}\right)_{n=1}^{m+1} \in \prod_{n=1}^{m+1} K_{n}:\left(x_{n}\right)_{n=1}^{m} \in U_{m} \text { and } x_{m+1} \in V_{m+1}\right\}
$$

clearly satisfies the desired condition. Setting now $U=\left\{x=\left(x_{n}\right)_{n \in \mathbb{N}} \in K\right.$ : $\left.\left(x_{n}\right)_{n=1}^{k} \in U_{k}\right\}$, we see that $U \cap F$ is non-empty and has $f$-diameter less than $\varepsilon$.

REMARK 3.3. The following proposition follows easily from the result of B. Cascales, E. Matoušková, I. Namioka and J. Orihuela mentioned in the Introduction, namely that a compact space is quasi Radon-Nikodým if and only if it is strongly fragmented. Here we give a proof based on Ribarska's characterization of fragmentability.

Proposition 3.4. A quasi Radon-Nikodým compact space is fragmented by a metric.

Before passing to the proof, recall Ribarska's characterization ([12], [8]).

A topological space $K$ is said to admit a separating $\sigma$-relatively open partitioning if there exists a family $\mathcal{U}=\bigcup_{n \in \mathbb{N}} \mathcal{U}_{n}$, each $\mathcal{U}_{n}=\left\{U_{n}^{\xi}: 0 \leq \xi \leq \xi_{n}\right\}$ being well ordered, such that:

1. Each $U_{n}^{\xi}$ is a relatively open subset of $K \backslash\left\{U_{n}^{\zeta}: \zeta<\xi\right\}$.

2. For each $n \in \mathbb{N}, \cup \mathcal{U}_{n}=K$.

3. For $x, y \in K$ and $x \neq y$, there exists $U \in \mathcal{U}$ such that $U \cap\{x, y\}$ is a singleton.

TheOREm 3.5 (Ribarska's characterization). A topological space $K$ is fragmentable if and only if it admits a separating $\sigma$-relatively open partitioning. 
Proof of Proposition 3.4. Let $K$ be a quasi Radon-Nikodým compact space and $f$ a lower semicontinuous fragmenting quasimetric on $K$. Define inductively

$$
\mathcal{U}_{n}=\left\{U_{n}^{\zeta}: \zeta \text { an ordinal number }\right\},
$$

letting $U_{n}^{\zeta}$ be a non-empty relatively open subset of $K \backslash \bigcup_{\eta<\zeta} U_{n}^{\eta}$ of $f$ diameter less than $1 / n$. The verification of Ribarska's characterization is routine.

The following theorem shows that the difference (if any) between the classes of Radon-Nikodým spaces and quasi Radon-Nikodým is very delicate. In fact they cannot be separated if we restrict them to totally disconnected compact spaces.

TheOREM 3.6. A totally disconnected quasi Radon-Nikodým compact space is a closed subset of a countable product of scattered compact spaces, and hence is Radon-Nikodým.

Proof. Let $L$ be a totally disconnected quasi Radon-Nikodým compact space and $f$ a lower semicontinuous fragmenting quasimetric on $L$. We may consider $L$ to be a subset of $\{0,1\}^{\Gamma}$ for some set $\Gamma$. For each $\gamma \in \Gamma$, set $V_{\gamma}^{0}=\pi_{\gamma}^{-1}(0)$ and $V_{\gamma}^{1}=\pi_{\gamma}^{-1}(1)$. Since $V_{\gamma}^{0}, V_{\gamma}^{1}$ are closed and disjoint subsets of $L$, Lemma 3.1 implies that there exists $n \in \mathbb{N}$ such that $f\left(V_{\gamma}^{0}, V_{\gamma}^{1}\right)>1 / n$. Thus if we set

$$
\Gamma_{n}=\left\{\gamma \in \Gamma: f\left(V_{\gamma}^{0}, V_{\gamma}^{1}\right)>1 / n\right\},
$$

then $\Gamma=\bigcup_{n \in \mathbb{N}} \Gamma_{n}$. Since $L$ is a closed subset of $\prod_{n \in \mathbb{N}} \pi_{\Gamma_{n}}(L)$, it suffices to prove that each $\pi_{\Gamma_{n}}(L)$ is scattered. So choose a closed subset $M$ of $\pi_{\Gamma_{n}}(L)$. By Zorn's Lemma there exists a closed subset $N$ of $L$ such that $\pi_{\Gamma_{n}} \mid N: N \rightarrow M$ is irreducible (i.e. it is onto $M$ and it is not onto $M$ when restricted to any closed subset of $N)$. In this case, if $V$ is a relatively open subset of $N$ of $f$-diameter less than $1 / n$, then $\pi_{\Gamma_{n}}(V)$ has non-empty interior in $M$. Since moreover, for every $\gamma \in \Gamma_{n}, f\left(\pi_{\gamma}^{-1}(0), \pi_{\gamma}^{-1}(1)\right)>1 / n$, it follows that

$$
\text { either } \quad V \cap \pi_{\gamma}^{-1}(0)=\emptyset \quad \text { or } \quad V \cap \pi_{\gamma}^{-1}(1)=\emptyset .
$$

Consequently, $\pi_{\Gamma_{n}}(V)$ is a single point in $\pi_{\Gamma_{n}}(L)$ and hence an isolated point of $M$.

Since a Radon-Nikodým compact space is also quasi Radon-Nikodým, we obtain:

Corollary 3.7 (E. Reznichenko). A totally disconnected Radon-Nikodým compact space is a closed subspace of a countable Cartesian product of scattered compact spaces. 
Since moreover a continuous image of a Radon-Nikodým compact space is quasi Radon-Nikodým, the following corollary solves the problem of continuous images in the case that they are also totally disconnected.

Corollary 3.8 (S. Argyros). A totally disconnected continuous image of a Radon-Nikodým compact space is Radon-Nikodým.

It is natural to ask at this point whether a lower semicontinuous fragmenting quasimetric characterizes a continuous image of a Radon-Nikodým compact. In this direction, we can prove the following:

Proposition 3.9. A compact space $K$ is the continuous image of a totally disconnected Radon-Nikodym compact if and only if it can be embedded in a compact space $K^{\prime}$ in such a way that there exists a lower semicontinuous quasimetric $f$ defined on $K^{\prime}$ and closed scattered subsets $L_{n}, n \in \mathbb{N}$, of $K^{\prime}$ such that each $L_{n}$ is $1 / n$-f-dense in $K^{\prime}$ (i.e. for each $x \in K^{\prime}$, there exists $y \in L_{n}$ such that $\left.f(x, y)<1 / n\right)$.

The above proposition easily yields an equivalent reformulation of the problem whether or not continuous images of totally disconnected RadonNikodým compact spaces are also Radon-Nikodým:

Proposition 3.10. The following are equivalent:

(i) Every continuous image of a totally disconnected Radon-Nikodým compact is Radon-Nikodým.

(ii) If $L$ is a compact space, $L_{n}$ closed scattered subspaces of $L$, and $f$ a lower semicontinuous quasimetric on $L$ such that each $L_{n}$ is $1 / n$ - $f$-dense in $L$, then $L$ is Radon-Nikodým.

Proof. If (i) is true and $L$ is as in (ii), then by Proposition 3.9, $L$ is the continuous image of a totally disconnected Radon-Nikodým compact space, being embeddable in itself. Hence it is Radon-Nikodým.

Conversely, if (ii) is true, and $L$ is the continuous image of a totally disconnected Radon-Nikodým compact space, then by the same proposition it is embeddable in a compact space $L^{\prime}$ which satisfies the conditions in (ii). Thus $L^{\prime}$ and therefore $L$ as a closed subset are Radon-Nikodým.

Proof of Proposition 3.9. For the "only if" part assume $L$ is a closed subset of $\prod_{n \in \mathbb{N}} M_{n}$ where each $M_{n}$ is scattered, and $\phi: L \rightarrow K$ is a continuous onto map. First we extend $\phi$ to all of $\prod_{n \in \mathbb{N}} M_{n}$ : Let $f_{i}: K \rightarrow[0,1]$ for $i \in I$ be continuous maps separating the points of $K$. For each $i \in I$ we can extend $f_{i} \phi$ to all of $\prod_{n \in \mathbb{N}} M_{n}$ by Tietze's Theorem. Let $\overline{f_{i} \phi}$ be this extension. Define now $K^{\prime} \subset[0,1]^{I}$ by

$$
x \in K^{\prime} \Leftrightarrow \exists y \in \prod_{n \in \mathbb{N}} M_{n}: x(i)=\overline{f_{i} \phi}(y) .
$$


It is easy to check that $K^{\prime}$ is closed, $K \subset K^{\prime}$ (as subsets of the cube $[0,1]^{I}$ ) and moreover the map $\bar{\phi}: \prod_{n \in \mathbb{N}} M_{n} \rightarrow K^{\prime}$ defined by $\bar{\phi}(y)(i)=\overline{f_{i} \phi}(y)$ is continuous and onto.

The map $d: \prod_{n \in \mathbb{N}} M_{n} \times \prod_{n \in \mathbb{N}} M_{n} \rightarrow[0,1]$ defined by $d(x, y)$

$= \begin{cases}1 /(\min \{n \in \mathbb{N}: x(n) \neq y(n)\}+1) & \text { if } \min \{n \in \mathbb{N}: x(n) \neq y(n)\}<\infty, \\ 0 & \text { otherwise, }\end{cases}$

is easily checked to be a lower semicontinuous fragmenting metric on $\prod_{n \in \mathbb{N}} M_{n}$. Since each $M_{n}$ is embeddable in the full product $\prod_{n \in \mathbb{N}} M_{n}$ we can set $L_{n}=\bar{\phi}\left(M_{n}\right)$. Clearly each $L_{n}$ is scattered as a continuous image of a scattered compact space. Thus, setting $f(x, y)=d\left(\bar{\phi}^{-1}(x), \bar{\phi}^{-1}(y)\right)$ for $x, y \in K^{\prime}$, we find as in Proposition 3.2 that $f$ is a lower semicontinuous fragmenting quasimetric, and it easily follows that each $L_{n}$ is $1 / n$ - $f$-dense in $K^{\prime}$.

For the "if" part, since it suffices to show that $K^{\prime}$ is a continuous image of a totally disconnected Radon-Nikodým compact space, we may assume that $K=K^{\prime}$.

Set $L=\prod_{n \in \mathbb{N}} L_{n}$ and define $h: L \rightarrow 2^{K}$ by

$$
h\left(\left(x_{n}\right)_{n \in \mathbb{N}}\right)=\bigcap_{n \in \mathbb{N}} B_{f}\left(x_{n}, 1 / n\right) .
$$

Claim 1. For each $x \in L, h(x)$ is either an empty set, or a singleton.

Assume $y_{1}, y_{2} \in h(x)$ with $y_{1} \neq y_{2}$. Since

$$
\bigcap_{n \in \mathbb{N}} B_{f}\left(y_{1}, 1 / n\right) \cap \bigcap_{n \in \mathbb{N}} B_{f}\left(y_{2}, 1 / n\right)=\left\{y_{1}\right\} \cap\left\{y_{2}\right\}=\emptyset
$$

and the sets are closed by the lower semicontinuity of $f$, there exists an $n_{0} \in \mathbb{N}$ such that $B_{f}\left(y_{1}, 1 / n_{0}\right) \cap B_{f}\left(y_{2}, 1 / n_{0}\right)=\emptyset$. Since moreover $y_{1}, y_{2} \in$ $B_{f}\left(\pi_{n_{0}}(x), 1 / n_{0}\right)$ we deduce that $\pi_{n_{0}}(x) \in B_{f}\left(y_{1}, 1 / n_{0}\right) \cap B_{f}\left(y_{2}, 1 / n_{0}\right)$, which is a contradiction.

Claim 1. The set $L_{\emptyset}=\{x \in L: h(x)=\emptyset\}$ is open in $L$.

Assume $x_{0} \in L$ and $h\left(x_{0}\right)=\emptyset$. Thus $\bigcap_{n \in \mathbb{N}} B_{f}\left(\pi_{n}\left(x_{0}\right), 1 / n\right)=\emptyset$. Consequently, there are $n_{1}, \ldots, n_{k}$ such that $\bigcap_{i=1}^{k} B_{f}\left(\pi_{n_{i}}\left(x_{0}\right), 1 / n_{i}\right)=\emptyset$. It is easy to see, using nets, that in this case there are open sets

$$
U_{n_{i}} \ni \pi_{n_{i}}\left(x_{0}\right) \quad \text { with } \bigcap_{i=1}^{k} B_{f}\left(y_{i}, 1 / n_{i}\right)=\emptyset \quad \text { for every } y_{i} \in U_{n_{i}} .
$$

Set $V_{n_{i}}=L_{n_{i}} \cap U_{n_{i}}$. Thus the set $V=\left\{x \in L: \pi_{n_{i}}(x) \in V_{n_{i}}, i=1, \ldots, k\right\}$ is open in $L$. Clearly $x_{0} \in V$ by (1). Let $x \in V$. Since then $\pi_{n_{i}}(x) \in V_{n_{i}} \subset U_{n_{i}}$, again by $(1), \bigcap_{i=1}^{k} B_{f}\left(\pi_{n_{i}}(x), 1 / n_{i}\right)=\emptyset$, and consequently $h(x)=\emptyset$. 
The set $L^{\prime}=L \backslash L_{\emptyset}$ is a closed subset of $L$ and thus a totally disconnected Radon-Nikodým compact space. Define $\phi: L^{\prime} \rightarrow K$ by $\phi(x) \in h(x)$. It remains to show that $\phi$ is continuous and onto.

Assume that $y \in K$ and find $x_{n} \in L_{n}$ such that $y \in B_{f}\left(x_{n}, 1 / n\right)$. Then $y \in \bigcap_{n \in \mathbb{N}} B_{f}\left(x_{n}, 1 / n\right)$, showing that $\phi$ is onto.

To show the continuity of $\phi$, let $\emptyset \neq U$ be an open subset of $K$ and $x \in \phi^{-1}(U)$. We will find a basic open $V$ such that $x \in V \subset \phi^{-1}(U)$. Set $M=K \backslash U$. Since $M$ is closed and $\phi(x) \notin M$, there exists $n_{1} \in \mathbb{N}$ such that $f(\phi(x), M)>1 / n_{1}$. Thus $B_{f}\left(M, 1 / n_{1}\right)$ is closed, contains $M$ and does not contain $\phi(x)$. Set

$$
V^{\prime}=K \backslash B_{f}\left(M, 1 / n_{1}\right) .
$$

Since $\bigcap_{n \in \mathbb{N}} B_{f}(\phi(x), 1 / n)=\{\phi(x)\} \subset V^{\prime}$, there exists $n_{2} \geq n_{1}$ such that

$$
B_{f}\left(\phi(x), 1 / n_{2}\right) \subset V^{\prime} .
$$

Set $V=\left\{y \in L: \pi_{n_{2}}(y) \in L_{n_{2}} \cap V^{\prime}\right\}$. To see that $x \in V$, observe that $\{\phi(x)\}=\bigcap_{n \in \mathbb{N}} B_{f}\left(\pi_{n}(x), 1 / n\right)$, thus $\pi_{n_{2}}(x) \in V^{\prime} \cap L_{n_{2}}$ by (3). By the definition of $V, x \in V$.

It remains to show that $\phi(V) \subset U$. Let $y \in V$. Thus $\pi_{n_{2}}(y) \in V^{\prime}$. According to $(2), f\left(\pi_{n_{2}}(y), M\right)>1 / n_{1} \geq 1 / n_{2}$. Thus $B_{f}\left(\pi_{n_{2}}(y), 1 / n_{2}\right) \cap M=\emptyset$, and since $\phi(y) \in B_{f}\left(\pi_{n_{2}}(y), 1 / n_{2}\right)$, it follows that $\phi(y) \notin M$. Since now $M=K \backslash U$, it follows that $\phi(y) \in U$, which is what we needed to prove.

4. Quasi Radon-Nikodým and Corson compact spaces. In this section we shall prove the following:

TheOrem 4.1. Let $K$ be a quasi Radon-Nikodým compact space. If $K$ is Corson compact, then it is already Eberlein.

Corollary 4.2 (J. Orihuela, W. Schachermayer, M. Valdivia, C. Stegall). A Radon-Nikodým Corson compact space is Eberlein.

Corollary 4.3 (C. Stegall). A continuous image of a Radon-Nikodým compact space is Eberlein provided that it is Corson.

Before passing to the proof of Theorem 4.1, we need first to define "derivative" sets in a quasi Radon-Nikodým compact space:

REMARK 4.1. Let $K$ be a quasi Radon-Nikodým compact space, and $f: K \times K \rightarrow[0,1]$ a lower semicontinuous fragmenting quasimetric on $K$. For $M$ a closed subset of $K$ and $n \in \mathbb{N}$, we define $\alpha_{n}(M)$ to be an ordinal number and $\beta_{n}(M)$ to be a closed subset of $M$ such that:

1. If $M_{2} \supset M_{1}$ then $\alpha_{n}\left(M_{2}\right) \geq \alpha_{n}\left(M_{1}\right)$. If moreover $\alpha_{n}\left(M_{2}\right)=\alpha_{n}\left(M_{1}\right)$ then $\beta_{n}\left(M_{2}\right) \supset \beta_{n}\left(M_{1}\right)$. 
2. $\beta_{n}(M)$ is covered by finitely many relatively open sets of $f$-diameter less than $1 / n$.

First we define the set $M^{(\alpha, n)}$ for a given ordinal $\alpha$ by induction:

- $M^{(0, n)}=M$.

- If $\alpha$ is a successor ordinal, then

$$
\begin{aligned}
M^{(\alpha, n)}=M^{(\alpha-1, n)} \backslash \bigcup\left\{U \subset M^{(\alpha-1, n)}: U\right. & \text { is relatively open } \\
& \text { and } f \text {-diam }(U)<1 / n\} .
\end{aligned}
$$

- If $\alpha$ is a limit ordinal, then $M^{(\alpha, n)}=\bigcap_{\beta<\alpha} M^{(\beta, n)}$.

Since every closed subset of $K$ has a relatively open subset of $f$-diameter less than $1 / n$, it follows that we can define the ordinal

$$
\gamma_{0}=\min \left\{\gamma: \gamma \text { ordinal and } M^{(\gamma, n)}=\emptyset\right\}
$$

It is not the case that $\gamma_{0}$ could be a limit ordinal, since then $\emptyset=M\left(\gamma_{0}, n\right)=$ $\bigcap_{\gamma<\gamma_{0}} M^{(\gamma, n)}$ and each $M^{(\gamma, n)}$ is closed and non-empty, which is a contradiction. Thus $\gamma_{0}$ is a successor ordinal and consequently

$$
\begin{aligned}
& M^{\left(\gamma_{0}-1, n\right)} \backslash \bigcup\left\{U \subset M^{\left(\gamma_{0}, n\right)}: U\right. \text { relatively open } \\
& \text { and } f \text {-diam }(U)<1 / n\}=\emptyset .
\end{aligned}
$$

Thus there exists a finite subcover of $M^{\left(\gamma_{0}, n\right)}$ by relatively open sets of $f$-diameter less than $1 / n$. We set

$$
\alpha_{n}(M)=\gamma_{0}-1 \quad \text { and } \quad \beta_{n}(M)=M^{\left(\alpha_{n}(M), n\right)} .
$$

It remains to show the first statement: Observe that for any ordinal $\alpha, M_{2}^{(\alpha, n)} \supset M_{1}^{(\alpha, n)}$. Thus $M_{2}^{\left(\alpha_{n}\left(M_{1}\right), n\right)} \supset M_{1}^{\left(\alpha_{n}\left(M_{1}\right), n\right)} \neq \emptyset$. Consequently, $\alpha_{n}\left(M_{2}\right) \geq \alpha_{n}\left(M_{1}\right)$. If $\alpha_{n}\left(M_{2}\right)=\alpha_{n}\left(M_{1}\right)$, then $\beta_{n}\left(M_{2}\right)=M_{2}^{\left(\alpha_{n}\left(M_{2}\right), n\right)} \supset$ $M_{1}^{\left(\alpha_{n}\left(M_{1}\right), n\right)}=\beta_{n}\left(M_{1}\right)$.

REMARK 4.2. Let $\left\{M_{k}\right\}_{k \in \mathbb{N}}$ be a sequence of closed subsets of $K$ such that $\bigcap_{k \in \mathbb{N}} M_{k} \neq \emptyset$. Then there exists $m \in \mathbb{N}$ such that $\beta_{n}\left(M_{1} \cap \ldots \cap M_{m}\right) \cap$ $M_{k} \neq \emptyset$ for all $k \geq m$.

For $m \in \mathbb{N}$, set $C_{m}=\bigcap_{r=1}^{m} M_{r}$. Since $\left\{C_{m}\right\}_{m \in \mathbb{N}}$ is a decreasing sequence of closed subsets, by Remark $4.1,\left(\alpha_{n}\left(C_{m}\right)\right)_{m \in \mathbb{N}}$ is a decreasing sequence of ordinals. Hence, there exists $m \in \mathbb{N}$ such that $\alpha_{n}\left(C_{k}\right)=\alpha_{n}\left(C_{m}\right)$ for all $k \geq m$. Then

$$
\begin{aligned}
\beta_{n}\left(C_{m}\right) \cap M_{k} & =C_{m}^{\left(\alpha_{n}\left(C_{m}\right), n\right)} \cap M_{k} \\
& \supset\left(C_{m} \cap M_{k}\right)^{\left(\alpha_{n}\left(C_{m}\right), n\right)} \supset C_{k}^{\left(\alpha_{n}\left(C_{k}\right), n\right)} \neq \emptyset .
\end{aligned}
$$

Before passing to the next lemma, let us recall some definitions: 
A family $\mathcal{U}$ of subsets of a topological space $K$ is called point countable (resp. point finite) if for every $x \in K$ the set $\{U \in \mathcal{U}: x \in U\}$ is countable (resp. finite).

$\mathcal{U}$ is called $\sigma$-point finite if it can be decomposed into countably many point finite subfamilies.

Also, it is called weakly separating if for every $x, y \in K$ with $x \neq y$ there exists $U \in \mathcal{U}$ such that $\{x, y\} \cap U$ is a singleton.

H. Rosenthal, using the characterization of D. Amir and J. Lindenstrauss for Eberlein compact spaces mentioned in the Introduction, has proved in [13] the following characterization:

TheOREm 4.4 (Rosenthal's characterization). A compact space $K$ is Eberlein compact if and only if it admits a $\sigma$-point finite weakly separating family of open $F_{\sigma}$ sets.

For convenience we use the following notation: A family $\left\{U^{\gamma}: \gamma \in \Gamma\right\}$ of sets over a set $\Gamma$ is also denoted by $\mathcal{U}^{(\Gamma)}$.

The following lemma gives a hint on how we are planning to prove that a Corson quasi Radon-Nikodým compact is Eberlein:

Lemma 4.5. Assume that $K$ is a quasi Radon-Nikodým compact, $f$ a lower semicontinuous fragmenting quasimetric on $K$, and $\mathcal{F}_{0}^{(\Gamma)}=\left\{F_{0}^{\gamma}: \gamma \in \Gamma\right\}$ and $\mathcal{F}_{1}^{(\Gamma)}=\left\{F_{1}^{\gamma}: \gamma \in \Gamma\right\}$ two families of closed sets of $K$ such that for each $\gamma \in \Gamma$,

$$
f\left(F_{0}^{\gamma}, F_{1}^{\gamma}\right)>1 / n
$$

If the family $\left\{K \backslash F_{0}^{\gamma}: \gamma \in \Gamma\right\}$ is point countable, then the family $\mathcal{F}_{1}^{(\Gamma)}$ is $\sigma$-point finite.

Note that condition (4) implies that $F_{1}^{\gamma} \subset K \backslash F_{0}^{\gamma}$.

Proof. We shall use induction on $|\Gamma|$, the cardinality of $\Gamma$.

For $\Gamma$ countable we have nothing to prove. Let $|\Gamma|>\aleph_{0}$ and assume that the statement holds for all cardinals less than $|\Gamma|$. Let $\Gamma=\left\{\gamma_{\alpha}: \alpha<|\Gamma|\right\}$ be an enumeration of $\Gamma$. For every $\alpha<|\Gamma|$ we define a set $\Gamma_{\alpha} \subset \Gamma$ such that $\bigcup_{\alpha<|\Gamma|} \Gamma_{\alpha}=\Gamma$ and $\left|\Gamma_{\alpha}\right|<|\Gamma|$, as follows:

- For a successor ordinal,

$$
\begin{aligned}
\Gamma_{\alpha}= & \left\{\gamma_{\beta}: \beta \leq \alpha\right\} \\
& \cup\left\{\gamma \in \Gamma: \exists \gamma_{1}, \ldots, \gamma_{k} \in \Gamma_{\alpha-1}: \beta_{n}\left(F_{1}^{\gamma_{1}} \cap \ldots \cap F_{1}^{\gamma_{k}}\right) \cap F_{1}^{\gamma} \neq \emptyset\right\} .
\end{aligned}
$$

- For a limit ordinal, $\Gamma_{\alpha}=\bigcup_{\beta<\alpha} \Gamma_{\beta}$.

Clearly $\Gamma=\bigcup_{\alpha<|\Gamma|} \Gamma_{\alpha}$. We shall show that $\left|\Gamma_{\alpha}\right| \leq \aleph_{0}|\alpha|$. This obviously holds for $\alpha=1$. It also holds if $\alpha$ is a limit ordinal, since $\left|\Gamma_{\beta}\right| \leq \aleph_{0}|\beta| \leq \aleph_{0}|\alpha|$ for all $\beta<\alpha$. 
Assume that $\alpha$ is a successor ordinal and $\left|\Gamma_{\alpha-1}\right| \leq \aleph_{0}|\alpha-1|$. Since the finite subsets of $\Gamma_{\alpha-1}$ are at most $\aleph_{0}|\alpha-1|$ many, it suffices to show that to each of them (say $\left\{\gamma_{1}, \ldots, \gamma_{k}\right\}$ ), there correspond countably many $\gamma \in \Gamma$ such that

$$
\beta_{n}\left(F_{1}^{\gamma_{1}} \cap \ldots \cap F_{1}^{\gamma_{k}}\right) \cap F_{1}^{\gamma} \neq \emptyset .
$$

By Remark 4.1, $\beta_{n}\left(F_{1}^{\gamma_{1}} \cap \ldots \cap F_{1}^{\gamma_{k}}\right)=\bigcup_{r=1}^{m} G_{r}$ with $f$-diam $\left(G_{r}\right)<1 / n$. Assuming that (5) holds for uncountably many $\gamma \in \Gamma$, there must be an $r \leq m$ such that $G_{r} \cap F_{1}^{\gamma} \neq \emptyset$ also holds for uncountably many $\gamma \in \Gamma$. Since $f$-diam $\left(G_{r}\right)<1 / n$ and $f\left(F_{1}^{\gamma}, F_{0}^{\gamma}\right)>1 / n$, for all those $\gamma$ 's it must be the case that $G_{r} \subset K \backslash F_{0}^{\gamma}$, which contradicts the assumption that $\left\{K \backslash F_{0}^{\gamma}: \gamma \in \Gamma\right\}$ is a point countable family.

Set now $\Delta_{\alpha}=\Gamma_{\alpha+1} \backslash \Gamma_{\alpha}$. Since $\left|\Delta_{\alpha}\right|<|\Gamma|$, by the inductive hypothesis there must exist $\Delta_{\alpha}^{n}$ for $n \in \mathbb{N}$ such that $\bigcup_{n \in \mathbb{N}} \Delta_{\alpha}^{n}=\Delta_{\alpha}$ and the family $\mathcal{F}_{1}^{\left(\Delta_{\alpha}^{n}\right)}$ is point finite. Set also $E_{n}=\bigcup_{\alpha<|\Gamma|} \Delta_{\alpha}^{n}$. Apparently $\Gamma=\bigcup_{n \in \mathbb{N}} E_{n}$. It suffices to show that each $\mathcal{F}_{1}^{\left(E_{n}\right)}$ is point finite. Since $\mathcal{F}_{1}^{\left(\Delta_{\alpha}^{n}\right)}$ is point finite, we just have to show that if $\gamma_{i} \in \Delta_{\alpha_{i}}$ with $\alpha_{1}<\alpha_{2}<\ldots$, then $\bigcap_{k \in \mathbb{N}} F_{1}^{\gamma_{k}}=\emptyset$. Assume not. By Remark 4.2 there exists $m \in \mathbb{N}$ such that $\beta_{n}\left(F_{1}^{\gamma_{1}} \cap \ldots \cap F_{1}^{\gamma_{m}}\right) \cap F_{1}^{\gamma_{k}} \neq \emptyset$ for all $k \geq m$. Since $F_{1}^{\gamma_{i}} \in \mathcal{F}_{1}^{\left(\Delta_{\alpha_{i}}\right)}$ and $\Delta_{\alpha_{i}}=\Gamma_{\alpha_{i}+1} \backslash \Gamma_{\alpha_{i}} \subset \Gamma_{\alpha_{i}+1} \subset \Gamma_{\alpha_{m}+1}$ we deduce by the definition of $\Gamma_{k}$ that $\gamma_{k} \in \Gamma_{\alpha_{m}+2}$ for all $k>m$ and thus $\gamma_{k} \in \Delta_{\alpha_{m}+1}$ for all $k>m$, which is a contradiction.

The following lemma generalizes Lemma 4.5.

Lemma 4.6. Assume that $K$ is a quasi Radon-Nikodým compact space, and $\mathcal{F}_{0}^{(\Gamma)}=\left\{F_{0}^{\gamma}: \gamma \in \Gamma\right\}$ and $\mathcal{F}_{1}^{(\Gamma)}=\left\{F_{1}^{\gamma}: \gamma \in \Gamma\right\}$ are two families of closed subsets of $K$ such that $F_{0}^{\gamma} \cap F_{1}^{\gamma}=\emptyset$ for each $\gamma \in \Gamma$. If the family $\left\{K \backslash F_{0}^{\gamma}: \gamma \in \Gamma\right\}$ is point countable, then the family $\mathcal{F}_{1}^{(\Gamma)}$ is $\sigma$-point finite.

Proof. This is immediate, since by Lemma 3.1 we can decompose $\Gamma=$ $\bigcup_{n \in \mathbb{N}} \Gamma_{n}$ in such a way that $f\left(F_{0}^{\gamma}, F_{1}^{\gamma}\right)>1 / n$ for each $\gamma \in \Gamma_{n}$ (where $f$ is the lower semicontinuous fragmenting quasimetric on $K)$.

Proof of Theorem 4.1. Let $f_{\delta}: K \rightarrow[0,1]$ for $\delta \in \Delta$ be continuous functions separating the points of $K$, such that for every $x \in K$ the set $\left\{\delta \in \Delta: f_{\delta}(x) \neq 0\right\}$ is countable. Let $\mathbb{Q} \cap(0,1]=\left\{q_{n}: n \in \mathbb{N}\right\}$ and set $\Gamma=\Delta \times \mathbb{N}$. Set also, for $\gamma=(\delta, n) \in \Gamma$,

$$
U_{1}^{\gamma}=f_{\delta}^{-1}\left(\left(q_{n}, 1\right]\right), \quad F_{0}^{\gamma}=f_{\delta}^{-1}(0), \quad F_{1}^{\gamma}=f_{\delta}^{-1}\left(\left[q_{n}, 1\right]\right) .
$$

Since $\left\{K \backslash F_{0}^{\gamma}: \gamma \in \Gamma\right\}$ is point countable and $F_{0}^{\gamma} \cap F_{1}^{\gamma}=\emptyset$ for each $\gamma \in \Gamma$, it follows by Lemma 4.6 that $\mathcal{F}_{1}^{(\Gamma)}$ is $\sigma$-point finite. Since moreover $U_{1}^{\gamma} \subset F_{1}^{\gamma}$ 
for each $\gamma \in \Gamma, \mathcal{U}_{1}^{(\Gamma)}$ is also $\sigma$-point finite and it is easy to check that it is moreover a weakly separating family consisting of open $F_{\sigma}$ sets.

By Rosenthal's characterization 4.4, the proof is complete.

\section{Quasi Radon-Nikodým spaces embeddable in $\mathcal{P}(S)$ for scat-} tered $S$. In what follows we shall denote by $\mathcal{P}(K)$ the space of regular Borel probability measures on $K$, endowed with the weak* topology.

We introduce a new class of compact spaces, the so-called almost totally disconnected spaces, which are a natural generalization of both totally disconnected and Corson spaces.

Definition 5.1. A compact space $K$ is called almost totally disconnected if it can be embedded in the cube $[0,1]^{\Gamma}$ in such a way that for each $x \in K$, the set $\{\gamma \in \Gamma: 0<x(\gamma)<1\}$ is countable.

Almost totally disconnected spaces are a strict generalization of totally disconnected and Corson spaces. Let us indicate an example that illustrates this:

The extended long line $L$ (see also [9]) is obtained from the ordinal space $\left[0, \omega_{1}\right]$ by inserting a copy of the interval $(0,1)$ between $\alpha$ and $\alpha+1$ for each ordinal $\alpha$ less than $\omega_{1}$.

For each $\alpha<\omega_{1}$ consider the map $f_{\alpha}: L \rightarrow[0,1]$ which is constantly 1 for $x \geq \alpha+1$, constantly 0 for $x \leq \alpha$ and linear between $\alpha$ and $\alpha+1$. It is easy to see that $\left\{f_{\alpha}: \alpha<\omega_{1}\right\}$ separates the points of $L$ and moreover for each $x \in L$ the cardinality of the set $\left\{\alpha<\omega_{1}: 0<f_{\alpha}(x)<1\right\}$ is at most one. Thus $L$ is almost totally disconnected. (In [9] it is proved that it is also Radon-Nikodým.) Clearly $L$ is not totally disconnected, and an application of the Pressing Down Lemma shows that it is not Corson.

We are going to prove the following:

THEOREM 5.1. An almost totally disconnected quasi Radon-Nikodým compact can be embedded in $\mathcal{P}(S)$ for some scattered compact $S$.

Since by [9, Theorem 2.5] for any Radon-Nikodým compact space $K$, $\mathcal{P}(K)$ is also Radon-Nikodým, we derive

Corollary 5.2. An almost totally disconnected compact space is Radon-Nikodým if and only if it is quasi Radon-Nikodým.

Since continuous images of Radon-Nikodým compact spaces are quasi Radon-Nikodým, we obtain

COROLlary 5.3. If a continuous image of a Radon-Nikodým compact is almost totally disconnected, then it is Radon-Nikodym.

In order to prove Theorem 5.1, we need first some technical lemmas: 
LemmA 5.4. Let $\mathcal{F}_{i}^{(\Gamma)}$ and $\mathcal{K}_{i}^{(\Gamma)}$ for $i=0,1$ be four families of closed subsets of a quasi Radon-Nikodým compact space $K$, over a countable set $\Gamma$. Let $f: K \times K \rightarrow[0,1]$ be a lower semicontinuous fragmenting quasimetric for $K$. Assume that there exists $m_{0} \in \mathbb{N}$ such that for all $\gamma \in \Gamma$,

$$
f\left(K \backslash\left(F_{0}^{\gamma} \cap F_{1}^{\gamma}\right), K_{0}^{\gamma} \cap K_{1}^{\gamma}\right)>1 / m_{0}, \quad f\left(\overline{K \backslash K_{0}^{\gamma}}, \overline{K \backslash K_{1}^{\gamma}}\right)>1 / m_{0},
$$

and the family $\mathcal{N}^{(\Gamma)}=\left\{N^{\gamma}=F_{0}^{\gamma} \cap F_{1}^{\gamma}: \gamma \in \Gamma\right\}$ is point finite. Set

$$
L=\left\{x \in\{0,1\}^{\Gamma}: \bigcap_{\gamma \in \Gamma} K_{x(\gamma)}^{\gamma} \neq \emptyset\right\} .
$$

Then $L$ is metrizable scattered, and therefore countable.

Proof. It is easy to see that $L$ is a closed subset of $\{0,1\}^{\Gamma}$. It suffices to prove that if $M \subset L$ is closed, then there exists an open subset $V$ of $L$ such that $V \cap M$ is non-empty and finite. For every $x \in M$ and every finite $A \subset \Gamma$ consider the ordinal number $\alpha(x, A)=\alpha_{m_{0}}\left(\bigcap_{\gamma \in A} K_{x(\gamma)}^{\gamma}\right)$ where $\alpha_{m_{0}}$ is the map of Remark 4.1. Let

$$
\alpha_{0}=\alpha\left(x_{0}, A_{0}\right)=\min \{\alpha(x, A): x \in M \text { and } A \subset \Gamma \text { finite }\}
$$

and set $V=\left\{x \in L: x(\gamma)=x_{0}(\gamma)\right.$ for all $\left.\gamma \in A_{0}\right\}$.

Clearly $V \cap M \neq \emptyset$. Set $F=\beta_{m_{0}}\left(\bigcap_{\gamma \in A_{0}} K_{x_{0}(\gamma)}^{\gamma}\right), \beta_{m_{0}}$ being the map of Remark 4.1. Let also $F=\bigcup_{r=1}^{\lambda} G_{r}$ with $f$-diam $\left(G_{r}\right)<1 / m_{0}$.

Claim 3. For every $x \in V \cap M$ and for every finite $B \supset A_{0}$,

$$
F \cap \bigcap_{\gamma \in B} K_{x(\gamma)}^{\gamma} \neq \emptyset
$$
Thus

Since $B \supset A_{0}$, we have $\bigcap_{\gamma \in B} K_{x(\gamma)}^{\gamma} \subset \bigcap_{\gamma \in A_{0}} K_{x(\gamma)}^{\gamma}=\bigcap_{\gamma \in A_{0}} K_{x_{0}(\gamma)}^{\gamma}$.

$$
\alpha_{m_{0}}\left(\bigcap_{\gamma \in B} K_{x(\gamma)}^{\gamma}\right) \leq \alpha_{m_{0}}\left(\bigcap_{\gamma \in A_{0}} K_{x_{0}(\gamma)}^{\gamma}\right)
$$

that is,

$$
\alpha(x, B) \leq \alpha\left(x_{0}, A_{0}\right)=\alpha_{0} .
$$

By the choice of $\alpha_{0}$ we have $\alpha(x, B)=\alpha_{0}$ and consequently

$$
\beta_{m_{0}}\left(\bigcap_{\gamma \in B} K_{x(\gamma)}^{\gamma}\right) \subset \beta_{m_{0}}\left(\bigcap_{\gamma \in A_{0}} K_{x_{0}(\gamma)}^{\gamma}\right)=F .
$$

Using (6) and the compactness of the sets involved in the intersection, we infer that $F \cap \bigcap_{\gamma \in \Gamma} K_{x(\gamma)}^{\gamma} \neq \emptyset$ for every $x \in V \cap M$. Assume now that $V \cap M$ is infinite. Since $F$ is a finite union of $G_{r}$ 's, there must exist $r_{0}$ such that the set

$$
M_{r_{0}}=\left\{x \in V \cap M: G_{r_{0}} \cap \bigcap_{\gamma \in \Gamma} K_{x(\gamma)}^{\gamma} \neq \emptyset\right\}
$$


is also infinite. Since the family $\mathcal{N}^{(\Gamma)}$ is point finite, there must exist a finite subset $B_{0}$ of $\Gamma$ such that $G_{r_{0}} \not \subset F_{0}^{\gamma} \cap F_{1}^{\gamma}$ for all $\gamma \in \Gamma \backslash B_{0}$. For each such $\gamma \in \Gamma \backslash B_{0}$,

$$
G_{r_{0}} \cap K_{0}^{\gamma} \cap K_{1}^{\gamma}=\emptyset
$$

since $f$-diam $\left(G_{r_{0}}\right)<1 / m_{0}$ and $f\left(\overline{K \backslash\left(F_{0}^{\gamma} \cap F_{1}^{\gamma}\right)}, K_{0}^{\gamma} \cap K_{1}^{\gamma}\right)>1 / m_{0}$. Also, since $f\left(\overline{K \backslash K_{0}^{\gamma}}, \overline{K \backslash K_{1}^{\gamma}}\right)>1 / m_{0}$ we find that

$$
G_{r_{0}} \cap K_{0}^{\gamma}=\emptyset \quad \text { or } \quad G_{r_{0}} \cap K_{1}^{\gamma}=\emptyset .
$$

Hence for each $\gamma \in \Gamma \backslash B_{0}$, we can define $y_{0}(\gamma) \in\{0,1\}$ such that $G_{r_{0}} \cap$ $K_{y_{0}(\gamma)}^{\gamma}=\emptyset$. Thus $x(\gamma) \neq y_{0}(\gamma)$ for each $x \in M_{r_{0}}$ and each $\gamma \in \Gamma \backslash B_{0}$. Since $B_{0}$ is a finite set we have arrived at a contradiction assuming that $M_{r_{0}}$ is infinite.

Lemma 5.5. Assume $K$ is a Hausdorff compact space and $\mathcal{K}_{\varepsilon}^{(\Gamma)}=\left\{K_{\varepsilon}^{\gamma}\right.$ : $\gamma \in \Gamma\}$ for $\varepsilon=0,1$ are two families of closed subsets of $K$ such that for each $\gamma \in \Gamma,\left(K_{0}^{\gamma}\right)^{\circ} \cup\left(K_{1}^{\gamma}\right)^{\circ}=K$, and for each $x \in\{0,1\}^{\Gamma}$, the cardinality of the set $\bigcap_{\gamma \in \Gamma} K_{x(\gamma}^{\gamma}$ is at most 1 . Let $L=\left\{x \in\{0,1\}^{\Gamma}: \bigcap_{\gamma \in \Gamma} K_{x(\gamma)}^{\gamma} \neq \emptyset\right\}$. Then $L$ is a closed subspace of $\{0,1\}^{\Gamma}$ and there exists an embedding of $K$ into $\mathcal{P}(L)$.

For a more detailed proof than the one presented here, we refer to [5].

Proof. It is easy to see that $L$ is indeed a closed subspace of $\{0,1\}^{\Gamma}$.

Using Urysohn's Lemma we define for each $\gamma \in \Gamma$ a continuous map $f_{\gamma}: K \rightarrow[0,1]$ such that $f_{\gamma} \mid K \backslash\left(K_{0}^{\gamma}\right)^{\mathrm{o}}=1$ and $f_{\gamma} \mid K \backslash\left(K_{1}^{\gamma}\right)^{\mathrm{o}}=0$. For each $k \in K$ and $\gamma \in \Gamma$ we can define a probability measure $p_{\gamma}(k)$ on $\{0,1\}$ with $p_{\gamma}(k)(\{1\})=f_{\gamma}(k)$ and $p_{\gamma}(k)(\{0\})=1-f_{\gamma}(k)$. Next we define the map

$$
u: K \ni k \mapsto \bigotimes_{\gamma \in \Gamma} p_{\gamma}(k) \in \mathcal{P}\left(\{0,1\}^{\Gamma}\right)
$$

where $\bigotimes_{\gamma \in \Gamma} p_{\gamma}(k)$ denotes the product measure of $p_{\gamma}(k)$. It can be checked that indeed $u(K) \subset \mathcal{P}(L)$ and since for each $x \in\{0,1\}^{\Gamma}, \bigcap_{\gamma \in \Gamma} K_{x(\gamma)}^{\gamma}$ is at most a singleton, $u$ is one-to-one.

Lemma 5.6. Let $S_{n}$ be Hausdorff compact spaces,

$$
L=\prod_{n \in \mathbb{N}} L_{n}, \quad S_{k}=\prod_{n=1}^{k} L_{n}
$$

and $S$ be the one-point compactification of the disjoint union of the $S_{k}$ 's. Then $\mathcal{P}(L)$ is embeddable in $\mathcal{P}(S)$.

Again, for a more detailed proof, we refer to [5]. 
Proof. We define a map $h: C(S) \rightarrow C(L)$ by

$$
h(f)\left(\left(\ell_{n}\right)_{n \in \mathbb{N}}\right)=\sum_{n=1}^{\infty} \frac{1}{2^{n}} f\left(\left(\ell_{k}\right)_{k=1}^{n}\right) .
$$

It is easy to verify that $h(f)$ is a continuous function on $\prod_{n \in \mathbb{N}} L_{n}$. Moreover, since $h$ is positive, $\|h\| \leq 1$ and $h\left(1_{S}\right)=1_{L}$, it follows that $h^{*}(\mathcal{P}(L)) \subset \mathcal{P}(S)$. It is also easy to see that $h(C(S))$ is norm dense in $C(L)$. Therefore $h^{*}$ is also one-to-one.

REMARK 5.1. Using the notation of the previous lemma, it is easy to check that if each $L_{n}$ is scattered, then so is $S$.

Lemma 5.7. Assume that $K$ is a quasi Radon-Nikodým compact space and $\mathcal{F}_{\varepsilon}^{(\Gamma)}=\left\{F_{\varepsilon}^{\gamma}: \gamma \in \Gamma\right\}$ and $\mathcal{K}_{\varepsilon}^{(\Gamma)}=\left\{K_{\varepsilon}^{(\Gamma)}: \gamma \in \Gamma\right\}$ for $\varepsilon=0,1$ are families of closed subsets of $K$ such that:

1. For each $x \in\{0,1\}^{\Gamma}$ the set $\bigcap_{\gamma \in \Gamma} K_{x(\gamma)}^{\gamma}$ is at most a singleton.

2. $\left(K_{0}^{\gamma}\right)^{\mathrm{o}} \cup\left(K_{1}^{\gamma}\right)^{\mathrm{o}}=K$ for all $\gamma \in \Gamma$.

3. The family $\mathcal{N}^{(\Gamma)}=\left\{N^{\gamma}=F_{0}^{\gamma} \cap F_{1}^{\gamma}: \gamma \in \Gamma\right\}$ is $\sigma$-point finite.

4. $K_{\varepsilon}^{\gamma} \subset\left(F_{\varepsilon}^{\gamma}\right)^{\circ}$ for all $\gamma \in \Gamma$ and $\varepsilon=0,1$.

Then there exists a totally disconnected Radon-Nikodým compact space $L$ such that $K$ is embeddable in $\mathcal{P}(L)$.

Proof. By Lemma 5.5, if we set

$$
L=\left\{x \in\{0,1\}^{\Gamma}: \bigcap_{\gamma \in \Gamma} K_{x(\gamma)}^{\gamma} \neq \emptyset\right\}
$$

then $K$ is embeddable in $\mathcal{P}(L)$.

It remains to show that $L$ is a closed subset of a countable product of scattered compact spaces.

Because of condition 2, $\left(\overline{K \backslash K_{0}^{\gamma}}\right) \cap\left(\overline{K \backslash K_{1}^{\gamma}}\right)=\emptyset$. Also, by condition 4, $K_{0}^{\gamma} \cap K_{1}^{\gamma} \subset\left(F_{0}^{\gamma} \cap F_{1}^{\gamma}\right)^{\circ}$ and hence $\left(\overline{K \backslash\left(F_{0}^{\gamma} \cap F_{1}^{\gamma}\right)}\right) \cap\left(K_{0}^{\gamma} \cap K_{1}^{\gamma}\right)=\emptyset$. By Lemma 3.1, for each $\gamma \in \Gamma$, there exists $m(\gamma) \in \mathbb{N}$ such that

$$
f\left(\overline{K \backslash K_{0}^{\gamma}}, \overline{K \backslash K_{1}^{\gamma}}\right)>\frac{1}{m(\gamma)}, \quad f\left(\overline{K \backslash\left(F_{0}^{\gamma} \cap F_{1}^{\gamma}\right)}, K_{0}^{\gamma} \cap K_{1}^{\gamma}\right)>\frac{1}{m(\gamma)} .
$$

Let $\Gamma=\bigcup_{n \in \mathbb{N}} \Gamma_{n}$ be such that each $\mathcal{N}^{\left(\Gamma_{n}\right)}$ is point finite, and define $\Gamma_{(n, m)}=$ $\left\{\gamma \in \Gamma_{n}: m(\gamma) \leq m\right\}$. It suffices to show that $\pi_{\Gamma(n, m)}(L)$ is scattered.

Let $A \subset \Gamma_{(n, m)}$ be countable. By Lemma 5.4, the set

$$
L \mid A=\left\{x \in\{0,1\}^{A}: \bigcap_{\gamma \in A} K_{x(\gamma)}^{\gamma} \neq \emptyset\right\}
$$

is countable and hence using a tree argument, we find that $L$ cannot contain any perfect set. 
Proof of Theorem 5.1. Let $\left\{x_{n}\right\}_{n \in \mathbb{N}}$ be an enumeration of the set $\left\{\left(q_{0}, q_{1}\right)\right.$ $\left.\in((0,1) \cap \mathbb{Q})^{2}: q_{0}>q_{1}\right\}$. For $n \in \mathbb{N}$ we find $\varepsilon(n)>0$ such that if $x_{n}=$ $\left(q_{0}, q_{1}\right)$ then $q_{0}-\varepsilon(n)>q_{1}+\varepsilon(n)$. Let moreover $f_{\delta}: K \rightarrow[0,1]$ for $\delta \in \Delta$ be a family of continuous maps separating the points of $K$, such that for all $x \in K,\left|\left\{\delta \in \Delta: f_{\delta}(x) \neq 0,1\right\}\right| \leq \aleph_{0}$. Set $\Gamma=\Delta \times \mathbb{N}$ and for given $\gamma=(\delta, n) \in \Gamma$ and $x_{n}=\left(q_{0}, q_{1}\right)$, define

$$
\begin{array}{ll}
F_{0}^{\gamma}=f_{\delta}^{-1}\left(\left[0, q_{0}\right]\right), & F_{1}^{\gamma}=f_{\delta}^{-1}\left(\left[q_{1}, 1\right]\right), \\
K_{0}^{\gamma}=f_{\delta}^{-1}\left(\left[0, q_{0}-\varepsilon(n)\right]\right), & K_{1}^{\gamma}=f_{\delta}^{-1}\left(\left[q_{1}+\varepsilon(n), 1\right]\right) .
\end{array}
$$

For the families $\mathcal{F}_{0}^{(\Gamma)}, \mathcal{F}_{1}^{(\Gamma)}, \mathcal{K}_{0}^{(\Gamma)}, \mathcal{K}_{1}^{(\Gamma)}$ we check the conditions of Lemma 5.7. Only condition 3 needs some verification:

For each $\gamma=(\delta, n) \in \Gamma$ set $N^{\gamma}=F_{0}^{\gamma} \cap F_{1}^{\gamma}$ and $M^{\gamma}=f_{\delta}^{-1}(\{0,1\})$. Then $N^{\gamma} \cap M^{\gamma}=\emptyset$ and by the hypothesis of the theorem, the family $\left\{K \backslash M^{\gamma}\right.$ : $\gamma \in \Gamma\}$ is point countable. Thus by Lemma 4.6 the family $\mathcal{N}^{(\Gamma)}$ is $\sigma$-point finite.

Thus there exists a totally disconnected Radon-Nikodým compact space $L$ such that $K$ is embeddable in $\mathcal{P}(L)$. Since in turn, by Remark $5.1, \mathcal{P}(L)$ is embeddable in the space of probability measures on some scattered compact space, the proof is complete.

We conclude this section with the following problem, posed by S. Argyros, which remains open:

Problem 1. Find whether every Radon-Nikodym compact space is embeddable in the space of probability measures on some scattered compact space.

\section{References}

[1] K. Alster, Some remarks on Eberlein compacts, Fund. Math. 104 (1979), 43-46.

[2] D. Amir and J. Lindenstrauss, The structure of weakly compact sets in Banach spaces, Ann. of Math. 88 (1968), 35-46.

[3] A. Arkhangel'skiı̌, General Topology II, Encyclopaedia Math. Sci. 50, Springer, 1996.

[4] S. Argyros, A note on Radon Nikodým compact sets, unpublished, 1996.

[5] S. Argyros and A. Arvanitakis, A characterization of regular averaging operators and its consequences, preprint, 2000.

[6] S. Ditor, On a lemma of Milutin concerning averaging operators in continuous function spaces, Trans. Amer. Math. Soc. 149 (1970), 443-452.

[7] M. Fabian, M. Heisler and E. Matoušková, Remarks on continuous images of RadonNikodým compacta, Comment. Math. Univ. Carolin. 39 (1998), 59-69.

[8] M. J. Fabian, Gâteaux Differentiability of Convex Functions and Topology, Wiley, 1997.

[9] I. Namioka, Radon-Nikodým compact spaces and fragmentability, Mathematika 34 (1987), 258-281. 
[10] J. Orihuela, W. Schachermayer and M. Valdivia, Every Radon-Nikodym Corson compact space is Eberlein compact, Studia Math. 98 (1991), 157-174.

[11] O. Reynov, On a class of Hausdorff compacts and GSG Banach spaces, ibid. 71 (1981), 113-126.

[12] N. Ribarska, Internal characterization of fragmentable spaces, Mathematika 34 (1987), 243-257.

[13] H. P. Rosenthal, The heredity problem for weakly compactly generated Banach spaces, Compositio Math. 28 (1974), 83-111.

[14] C. Stegall, More facts about conjugate Banach spaces with the Radon-Nikodym property II, Acta Univ. Carolin. Math. Phys. 32 (1991), 47-54.

Department of Mathematics

University of Athens

15784 Panepistimiopolis, Athens, Greece

E-mail: aarvan@cc.uoa.gr

Received 5 December 2000;

in revised form 13 July 2001 